\title{
Saudi Medical Journal (2018) continues upswing
}

The year 2019 marks an important turning point in the journal's history as we celebrate our 4 decades of success. Since its inception in 1979, Saudi Medical Journal (SMJ) has expanded and progressed fast with continuous growth. Saudi Medical Journal publish variety of medical articles of variable content like local Clinical Guidelines, Systematic Review, Original article, Case Report, and Brief Report. Saudi Medical Journal encourage interaction with our readers and we publish valuable Correspondence material. The key to our success lies not merely in the well-known indicators or advanced systems but its leadership and people. It is the heart of SMJ who truly embodies the mission and vision of the journal. We could not serve successfully without their commitments and dedications. We take pride as we embark on the next decade of success along with several significant plans and initiatives to reflect our global presence and respected reputation.

For the second consecutive year, SMJ received a high Journal Impact Factor (JIF), standing at 1.055 from last year's 0.709 (Figure 1). We are pleased with the journal's growing number of citation impact, influence and reach. 2018 has again been a year with remarkable achievements. We would like to express sincere appreciation for the enthusiastic and vigorous support from all members of the editorial team, our valued readers, authors and reviewers whose significant contributions helped to maintain the standards of the journal through the years. A sustainable JIF standing is dependent on visibility and indexing in renowned scholarly databases. Considering this fact, we are continuously striving to improve and enhance the quality and reputation of this journal, and we are pleased to announce the indexing of SMJ in ProQuest Database.
This year, we welcomed submissions of new sections such as Protocol for Systematic Review and Video as a Supplementary file to an article. Systematic Review Protocol should be registered in PROSPERO registry. ${ }^{1}$ The author must disclose ethical approval and funding from a recognized body. Funded studies will be peerreviewed by the assigned editor while non-funded research will undergo external peer review. The author should refer to the PRISMA-P Checklist to facilitate the reporting of Systematic Review Protocol. ${ }^{2}$ By applying advanced technologies to make content discoverable and accelerate the path to insight, knowledge and better decision-making, there have been growing interest among publishers and journals to use video as supplementary material to publications. Video materials are effective visual communication mediums to highlight and enhance the value of a publication. While still images are valuable, we would like to welcome this innovation in our future publications. Authors may include still images or videos, or both materials in their article. All submitted videos will be peer-reviewed by the editorial board. Necessary permission and consent for a video material to be published is mandatory and should be provided during submission. Detailed requirements for these sections will be available in the "Instructions to Author" for reference. It is understood that these additions will enrich the journal's content.

In April 1 2018, we organized the 3rd Medical Publication course. The course was designed for small groups and led by our wide range of extensive experience editors, speakers were invited and few members of the editorial staff participated in the workshop. The sessions included structured exercises, stimulating collaborative thinking discussions, question and answer, sharing of ideas and experiences to gain expert knowledge

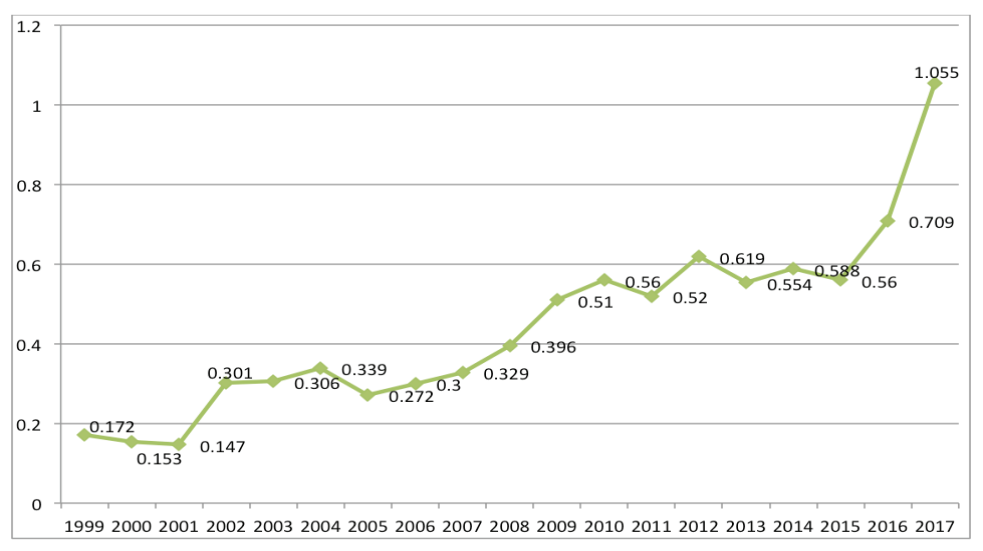

Figure 1 - SMJ Journal Impact Factor from 1999-2017. 


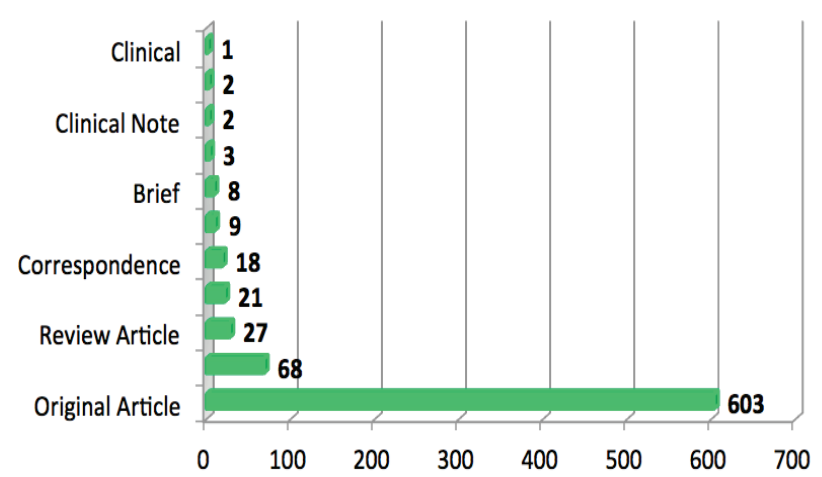

Figure 2 - Type of manuscripts received for the year 2018.

and networking with others. Overall it was indeed a productive and successful workshop and we received very overwhelming feedback from the participants.

We have weathered many changes and challenges in the past but we remained strong and true to our objective. It is our great honor to introduce the operational highlight for the third quarter of this year; the launch of our new online submission and peer review management system. Now, all submissions should be tendered via ScholarOne. ${ }^{3}$ ScholarOne will automate repetitive processes and eliminate redundant tasks. With this, we anticipate a high level of efficiency and productivity in our daily routine of toil. It is one step to our continuous commitment to improve the editorial processes and better serve our authors and reviewers. Continual system investments as such ensure stability, security and relevance. Simultaneously, we are assessing other journal system requirements and our future plan is to move to a new website hosting company to expand the scope of our journal.

Annual statistics. This year we received 762 manuscripts from which we processed 361 articles that have complied with the journal requirements (Figure 2). Over the past 12 issues, we have published 2 Editorials, 5 Systematic Reviews, and 128 Originals, with a total of 1161 pages. Sixty-one percent of the papers we published were from the Kingdom of Saudi Arabia (Table 1). Our total rejection rate was $75 \%$ of which $53 \%$ were rejected at the initial decision. The average processing time frame of original articles in the year 2018 from received date to acceptance was 2.4 months, from acceptance to publication 1.5 months, and from received to publication 3.9 months.

We are grateful for the invaluable contribution of our board members. We are pleased to announce the following new additions to our Editorial Team: Emad
Table 1 - Origin of "peer-reviewed" articles published in the Saudi Medical Journal for the year 2018.

\begin{tabular}{lrr}
\hline Origin & $\mathbf{n}$ & $(\%)$ \\
\hline Kingdom of Saudi Arabia & 120 & $(61.2)$ \\
Gulf countries & & \\
United Arab Emirates & 2 & $(1.0)$ \\
Bahrain & 1 & $(0.5)$ \\
Arab countries/Eastern Mediterranean Region & & \\
$\quad$ Iraq & 11 & $(5.6)$ \\
Jordan & 4 & $(2.0)$ \\
Iran & 0 & \\
Egypt & 5 & $(2.6)$ \\
Others & & \\
Karakhastan & 1 & $(0.5)$ \\
Turkey & 29 & $(14.8)$ \\
United Kingdom & 3 & $(1.5)$ \\
China & 8 & $(4.1)$ \\
Indonesia & 2 & $(1.0)$ \\
Korea & 4 & $(2.0)$ \\
Italy & 1 & $(0.5)$ \\
Ecuador & 1 & $(0.5)$ \\
Malaysia & 1 & $(0.5)$ \\
Brunei & 1 & $(0.5)$ \\
Sudan & 1 & $(0.5)$ \\
Pakistan & 1 & $(0.5)$ \\
Total & 196 & $(100.0)$ \\
\hline
\end{tabular}

Raddaoui, Mohamed Zamzam, Hani Mawardi, Pinar Okyay, Waleed Awwad, and Nasser Al Asseri. Also, we bid farewell to Manal Al Sheddi, Abdullah Alabassi, Fayek Al-Hilli, Abimbola Osoba, Meiyan Jiang, Sabah Jastaneiah, Ohoud Owaidhah, Samira Awaji, and Pippa Smart. We are grateful for their outstanding commitment and invaluable contribution and wish them success in all of their future endeavors. We would like to take this opportunity to thank our editors who have tried their best to find qualified reviewers and deliver timely editorial decisions.

Again we would like to thank everyone who supported us through the year. On behalf of the Journal, we wish you success!

\section{References}

1. Centre for Reviews and Dissemination. PROSPERO: International prospective register of systematic reviews. (Update 2011; Accessed 2018 December 3). Available from: https:// www.crd.york.ac.uk/prospero/

2. The PRISMA Group. Preferred Reporting Items for Systematic Reviews and Meta-Analyses. [Update 2009; Accessed 2018 December 3]. Available from: http://www.prisma-statement. org/Extensions/Protocols.aspx

3. Clarivate Analytics. ScholarOne, Inc. [Update 2018; Accessed 2018 December 3]. Available from: https://mc04. manuscriptcentral.com/psmmc-smj

\section{Fahdah Alokaily, MD Editor-in-Chief}

